\title{
Pacemaker Syndrome During Managed Ventricular Pacing Mode: What is the Mechanism?
}

\author{
PATRIZIO PASCALE, M.D., ETIENNE PRUVOT, M.D., and DENIS GRAF, M.D. \\ From the Service of Cardiology, Centre Hospitalier Universitaire Vaudois and University of Lausanne, Lausanne, Switzerland
}

\section{Case Presentation}

A 51-year-old woman underwent implantation of a dual chamber pacemaker 10 years ago, for symptomatic intermittent second-degree type II AV block. She remained free of symptoms during follow-up. Recently, she underwent pacemaker replacement because of battery depletion (Adapta ADDR01, Medtronic Inc., Minneapolis, MN, USA). Given intact AV conduction at the time of intervention, the Managed Ventricular Pacing mode algorithm (MVP ${ }^{\mathrm{TM}}$, Medtronic), which provides $\mathrm{AAI}(\mathrm{R})$ pacing with ventricular monitoring and backup DDD/R pacing as needed, was programmed with a lower rate limit of $60 \mathrm{ppm}$ and an upper rate limit of $130 \mathrm{ppm}$.

Ten days after the procedure, the patient complained of asthenia, dyspnea, and presyncope. ECG at pacemaker interrogation revealed sinus tachycardia with second-degree type II AV block (Fig. 1). Atrial pacing artifacts with atrial capture are seen dissociated from the native QRS complexes. Pacemaker interrogation revealed pacing and sensing thresholds as well as impedances within normal range. In our patient, the repetitive atrial pacing during ventricular systole led to pacemaker syndrome and symptoms resolved by reprogramming the device to AV sequential pacing without MVP. What is the mechanism of atrial stimulation dissociated from the native QRS complexes?

\section{Discussion}

Pacemaker ECG with marker annotation (Fig. 2A) showed that dissociated atrial pacing stimuli were related to functional undersensing, with atrial native beats falling within the refractory period after sensed or paced atrial beats, preventing the resetting of the atrial timer as the pacemaker operated in AAI mode. At the end of the atrial lower rate interval, atrial stimulation is delivered dissociated from the native QRS complexes. This case illustrates a particular cause of pacemaker syndrome resulting from two related mechanisms: (1) functional undersensing of sinus beat related to the atrial refractory period operated by the device during MVP mode and (2) failure of the unit to switch from AAI (R) to

J Cardiovasc Electrophysiol, Vol. 20, pp. 574-576, May 2009.

Address for correspondence: Patrizio Pascale, M.D., Service of Cardiology, Centre Hospitalier Universitaire, Vaudois-BH07, 1011 Lausanne, Switzerland. Fax: +41213140013; E-mail: Patrizio.Pascale@ chuv.ch

doi: $10.1111 / \mathrm{j} .1540-8167.2008 .01398 . x$
DDD (R) pacing mode despite a second-degree type II AV block. During MVP, the AAI $(R)$ mode operates with a dynamic atrial refractory period (ARP) set either to $75 \%$ of the $\mathrm{R}-\mathrm{R}$ cycle length if heart rate is $75 \mathrm{bpm}$ or faster, or to $600 \mathrm{~ms}$ if heart rate is slower than $75 \mathrm{bpm}$. The purpose of dynamic ARP is to avoid inappropriate switches to $\mathrm{DDD}(\mathrm{R})$ mode in the presence of (nonconducted) premature atrial contractions and to ensure that the atrial escape interval is only reset after true $\mathrm{P}$ waves. When the device is operating in MVP mode, ventricular pacing may be delivered in case of intermittent or transient loss of AV conduction. This backup ventricular pacing is scheduled to occur after any $\mathrm{A}-\mathrm{A}$ interval in which there is no ventricular sensed event, and occurs $80 \mathrm{~ms}$ after the scheduled atrial pace (i.e., $80 \mathrm{~ms}$ after the escape A-A interval). MVP switches the device from AAI(R) to DDD(R) when there is evidence of persistent loss of AV conduction defined as the absence of ventricular sensed event for 2 out of the last 4 pacing cycles (the four most recent A-A intervals). This aims to prevent inappropriate switches following single nonconducted atrial events.

As illustrated on the ECG (Fig. 2B), native sinus beats repetitively fall in the ARP since the device operates a refractory period based on the $\mathrm{R}-\mathrm{R}$ cycle length rather than on the A-A interval, which is half shorter. Two consequences derive from this condition: first, refractory sinus beats do not reset the timer of atrial escape interval $(1000 \mathrm{~ms})$ and, as a consequence, pacing stimuli are delivered dissociated from the QRS complexes. Second, repetitive sinus beats falling in the ARP provide the condition for failure to switch to $\mathrm{DDD}(\mathrm{R})$ mode since ventricular sensed events persistently occur during each pacemaker A-A interval (the atrial escape interval instead of the sinus beat interval) despite seconddegree type II AV block. A similar inappropriate mode of operation is shown in another patient with sinus tachycardia and second-degree type I 4:3 AV block (Fig. 3).

\section{Conclusion}

With an increased understanding of the potential detrimental effects of right ventricular pacing, ${ }^{1-4}$ new pacing algorithms such as the MVP mode have been developed to minimize ventricular pacing. This case illustrates that MVP feature may potentially lead to pacemaker syndrome in patients with second-degree AV block. The randomized studies of MVP to date have been mainly restricted to patients with intact AV conduction with only about $6 \%$ of the enrolled patients with previously documented AV block. 3,5,6 Therefore, we have little knowledge of the actual clinical impact 


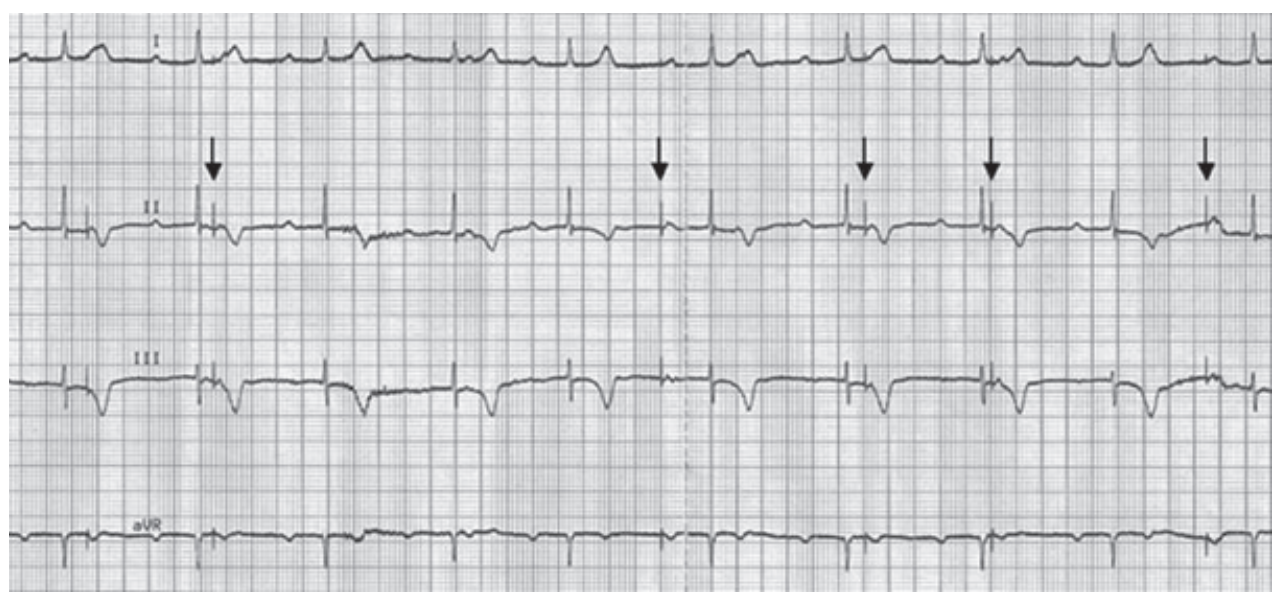

Figure 1. ECG 10 days after pacemaker replacement. Note the atrial pacing artifacts (arrows) dissociated from the native QRS complexes.
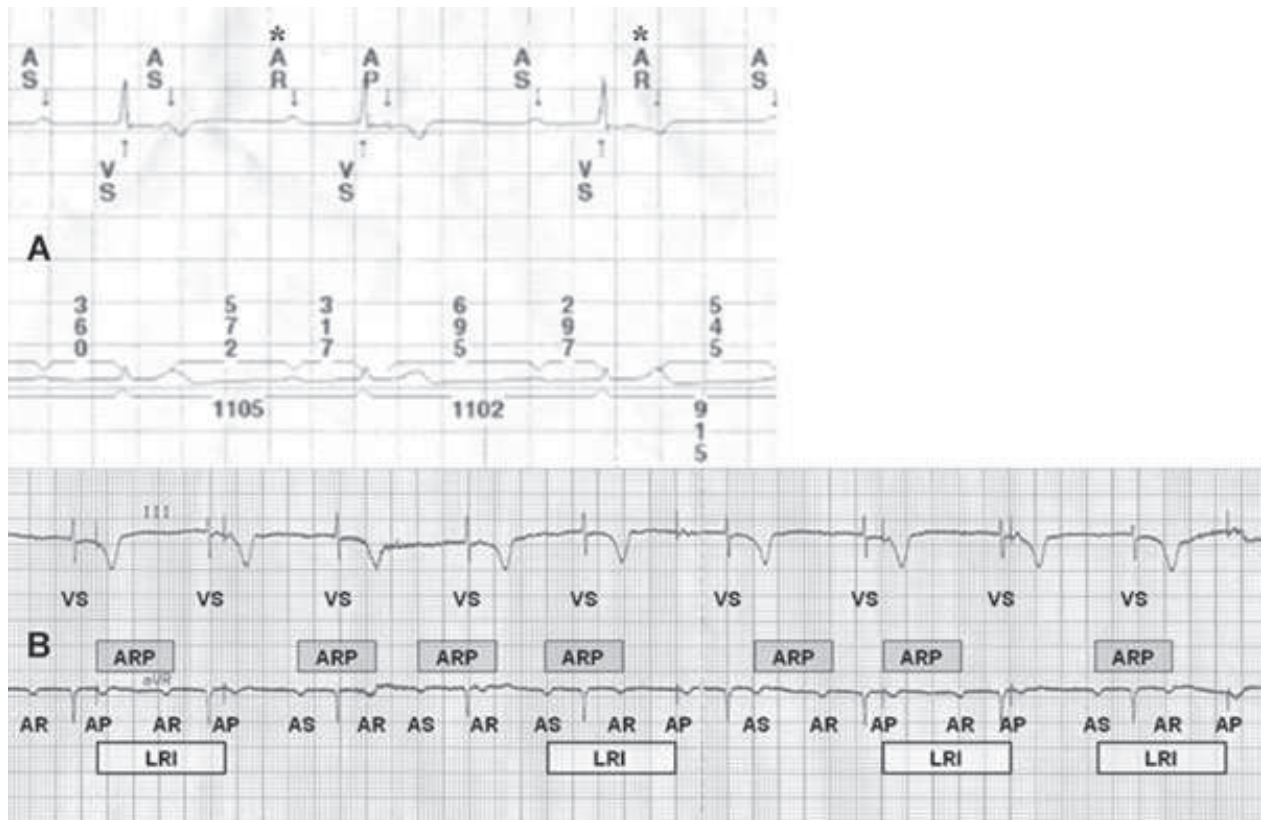

Figure 2. A: Pacemaker ECG with marker annotation during MVP operation. Note the atrial pacing stimulus (AP) following functional undersensing of atrial sinus beats falling within the refractory period $\left(A R^{*}\right)$ triggered by sensed atrial beats (AS), preventing the resetting of the atrial timer as the pacemaker operates in AAI mode. B: ECG with marker annotation and timing of the atrial refractory period (ARP) and lower rate interval (LRI) operated by the device illustrating the aforementioned observations. Note that the functionally undersensed sinus beats (AR) falling in the ARP provide the condition for failure to switch to DDD $R$ ) mode, since ventricular sensed events (VS) persistently occur between two consecutive atrial sensed or paced events.

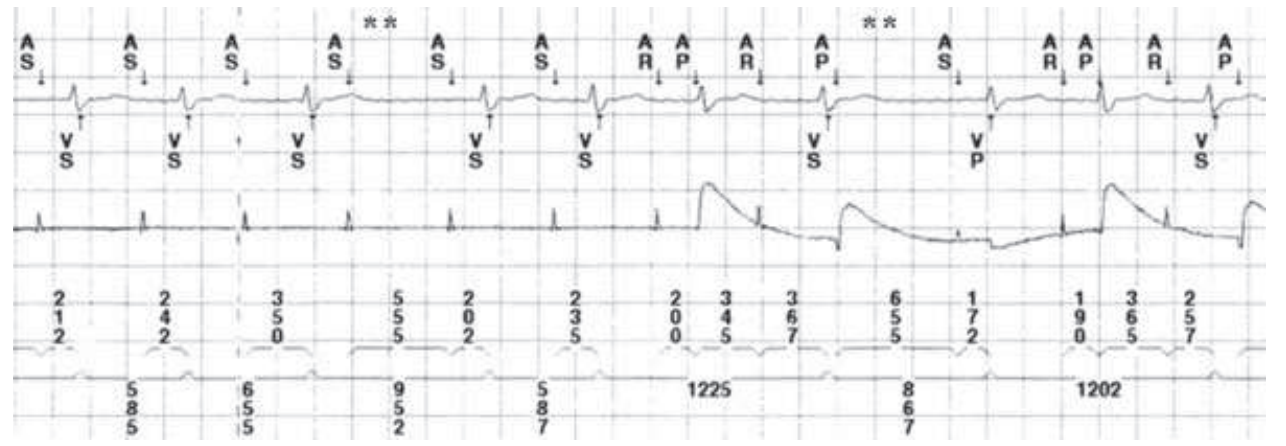

Figure 3. ECG and stored electrogram with marker annotation in a patient with sinus tachycardia and second-degree type I 4:3 AV block. A similar inappropriate mode of operation is illustrated with sinus beats falling within the atrial refractory period (AR) with atrial pacing artifacts (AP) dissociated from the native QRS (VS). The pacemaker does not switch to $D D D(R)$ mode since, except on two separate occasions $\left(^{* *}\right)$, ventricular sensed events always occur between two consecutive atrial sensed or paced events. 
of this inappropriate mode of operation, and careful consideration should be given to this potential drawback in patients with intermittent second-degree AV block or who develop symptoms suggestive of pacemaker syndrome during MVP mode.

Since the atrial refractoy period operated by the device during MVP mode is a nonprogrammable feature, the only programming option is to inactivate the MVP mode and, if desired, to program the Search AV + feature to promote intrinsic ventricular activation in the event of restored AV conduction and to prevent inappropriate long AV delay if AV block persists.

\section{References}

1. Gillis AM, Chung MK: Pacing the right ventricle: To pace or not to pace? Heart Rhythm 2005;2:201-206.

2. Sweeney MO, Hellkamp AS, Ellenbogen KA, Greenspon AJ, Freedman RA, Lee KL, Lamas GA, for the MOde Selection Trial (MOST) Investigators: Adverse effect of ventricular pacing on heart failure and atrial fibrillation among patients with normal baseline QRS duration in a clinical trial of pacemaker therapy for sinus node dysfunction. Circulation 2003;107:2932-2937.

3. Sweeney MO, Bank AJ, Nsah E, Koullick M, Zeng QC, Hettrick D, Sheldon T, Lamas GA: Search AV extension and managed ventricular pacing for promoting atrioventricular conduction (SAVE PACe) trial. $\mathrm{N}$ Engl J Med 2007;357:1000-1008.

4. Wilkoff BL, Cook JR, Epstein AE, Green HL, Hallstrom AP, Hsia H, Kutalek SP, Sharma A, Dual chamber and VVI Implantable Defibrillator Trial Investigators: Dual-chamber pacing or ventricular backup pacing in patients with an implantable defibrillator. The dual chamber and VVI implantable defibrillator (DAVID) Trial. JAMA 2002;288: 3115-3123.

5. Gillis AM, Pürerfellner H, Israel CW, Sunthorn H, Kacet S, Anelli-Monti M, Tang F, Young M, Boriani, on behalf of the Medtronic Enrhythm Clinical Study Investigators: Reducing unnecessary right ventricular pacing with the managed ventricular pacing mode in patients with sinus node disease and AV block. Pacing Clin Electrophysiol 2006;29:697-705.

6. Sweeney MO, Ellenbogen KA, Casavant D, Betzold R, Sheldon T, Tang F, Mueller M, Lingle J, for the Marquis MVP Download Investigators: Multicenter, prospective, randomized safety and efficacy study of a new atrial-based managed ventricular pacing mode (MVP) in dual chamber ICDs. J Cardiovasc Electrophysiol 2005;16:811-817. 